\title{
Congestion Tolls as Utility Alignment Between Agent and System Optimum
}

\author{
Ana L. C. Bazzan* and Robert Junges \\ Instituto de Informática, UFRGS \\ Caixa Postal 15064 \\ 91.501-970 Porto Alegre, RS, Brazil \\ \{bazzan,junges\}@inf.ufrgs.br
}

\begin{abstract}
In multi-agent systems, greedly agents can harm the performance of the overall system. This is the case of traffic commuting scenarios: drivers repete their actions trying to adapt to daily changes. In this domain, there are several proposals to achieve the traffic network equilibrium. Recently, the focus has shifted to information provision in several forms as a way to balance the load. Most of these works make strong assumptions such as the traffic authority and/or drivers having perfect information. In reality, the information the central control provides to drivers contains estimation errors. The goal of this paper is to propose a socially efficient load balance by internalizing social costs caused by agents' actions. Two issues are addressed: the model of information provision accounts for information imperfectness, and the equilibrium which emerges out of drivers route choices is close to the system optimum due to mechanisms of road pricing. The model can then be used for traffic authorities to simulate the effects of information provision and toll charging.
\end{abstract}

\section{Categories and Subject Descriptors}

I.2.11 [Artificial Intelligence]: Distributed Artificial Intelligence

Multiagent systems, Coherence and coordination

\section{General Terms}

Economics

\section{INTRODUCTION}

The increasing demand for mobility in our society causes frequent jams in highways. Control measures have been proposed to better control the utilization of the available infra-structure; among these we find driver information and guidance systems (DIGS). Normally, information provision

${ }^{*}$ Author supported by $\mathrm{CNPq}$

Permission to make digital or hard copies of all or part of this work for personal or classroom use is granted without fee provided that copies are not made or distributed for profit or commercial advantage and that copies bear this notice and the full citation on the first page. To copy otherwise, to republish, to post on servers or to redistribute to lists, requires prior specific permission and/or a fee.

AAMAS'06 May 8-12 2006, Hakodate, Hokkaido, Japan.

Copyright 2006 ACM 1-59593-303-4/06/0005 ...\$5.00. and route guidance strategies aim at achieving the system optimum (minimization or maximization of some global objective criterion). Note that this does not imply the user optimum and vice-versa. User optimum means equal costs for all alternative routes connecting two points in the network, for each road user. This may lead to system' suboptimality. If the focus is on global optimum, then the route guidance system may eventually recommend a route which is more costly (for a single user) than it would be the case if the user optimum were to be recommended. In general, traffic control authorities are interested in the system optimum, while the user seeks its own optimum.

In a more general way, this question relates to emergence of coordination in multiagent systems, in cases where the agents do not communicate and thus have to achieve coordination in a somehow greedy way. This normally causes the system to perform suboptimally. Therefore, it is important to align the user and the system optimum. Previous works have been proposed but mostly they assume some degree of cooperation and communication among the agents. Since drivers are self-interested, this alignment cannot be imposed by the system as it has been proposed in other works.

\section{RELATED WORK: TRAFFIC, INFORMA- TION, AND COLLECTIVES}

Modeling traffic scenarios with multi-agent systems techniques is not new. However, the focus has been mainly on coarse-grained level regarding traffic problems as traffic agents monitoring problem areas. On the other hand, our long term work focuses on a fine-grained level or rather on traffic flow control.

Our previous work with a scenario of iterated route choice provides a good opportunity to test learning and emergence of coordination. The main problem is to achieve system optimum or at least acceptable patterns of traffic distribution out of user's own performance strategies, i.e. users trying to adapt to the traffic patterns they experiment every day. There are different means to achieve a certain alignment of system and user optimum without relegating important issues such as lack of knowledge of non-commuter users, traffic information and forecast, etc.

The case of simple user adaptation in a binary route choice scenario, with and without traffic forecast was tackled in [3]. A scenario was simulated where $N$ drivers had to decide for one out of the available routes, in every round. At the end of the round, every driver gets a reward that is computed based on the number of drivers who selected the same route, 
in a kind of coordination game. In [4] different forms of traffic information - with different associated costs - were analyzed which may be used to assess the future traffic situation for the selection of one route. Finally, information recommendation and manipulation was tested in [2] with a traffic control center giving manipulated information for drivers in the scenario of the Braess Paradox, in a tentative of diverting drivers to less congested routes.

In all these works, different means of utility alignment were tried. Some were more successful than others regarding the performance of the global metrics. However, in the present paper we want to drop the "perfect information" assumption (both the traffic control center and all individuals having knowledge of all alternatives).

From the perspective of the economics of traffic, road pricing has been proposed as a way to realize efficient road utilization i.e. to achieve a distribution of traffic volume as close as possible to the system optimum. Congestion toll is one of the road pricing methods: considering the system optimum, a toll is computed which is the difference between the marginal social cost and the marginal private cost. Notice that this difference can be negative meaning that drivers actually get a reimbursement.

Regarding the future traffic situation as well as the effects of the toll system (and other measures to control the traffic), most of the work published has assumed that the control center has perfect information, meaning that it will know precisely the states of near future traffic conditions. This is a hard assumption given that acurate weather and accident forecasts are not possible. A state-dependent toll pricing system is discussed in [5]. Additionally, it is investigated which are the impacts of two alternatives toll schemas: toll is charged before or after drivers select a route. This distinction is important because congestion tolls, when announced before the drivers make their decisions, carry some meaningful information.

Still related to the quest of user vs. system optimum, Tumer and Wolpert [6] have investigated the use of a multiagent system to control routing of packages in a computer network, as well as the sensitivity of the network to the Braess paradox using the Collective Intelligence (COIN) formalism. In COIN, the world utility, $G(\zeta)$, is an arbitrary function of the state of all agents across all times. The utility for an agent is given by the difference between the total cost accrued by all agents in the network and the cost accrued by agents when all agents sharing the same destination are "erased". The problem with the use of this approach in a road network is that we cannot expect the agents (drivers) to have any idea about the global goal or cost. This is possible in the computer network scenario because the agents are router nodes which have knowledge about the throughput (packages to be routed to different destinations).

\section{MODEL AND RESULTS}

We have developed a simple model for the adaptive route choice. Since an agent has only qualitative information about the routes, and none about the other agents, each agent needs an expectation of the costs $\mathrm{s} /$ he will have if $\mathrm{s} / \mathrm{he}$ selects a certain route. This can be understood as the probability according to which a driver selects one route. For instance, if it is 1 for route $r$ then the driver always takes route $r$. With a certain periodicity, driver $d$ updates this heuristic according to the rewards $\mathrm{s} /$ he has obtained on the routes $\mathrm{s} /$ he has taken so far. The update of the heuristic is done according to the following formula: heuristics $\left(d, r_{i}\right)=$ $\frac{\sum_{t} \text { utility }_{r_{i}}(d, t)}{\sum_{i} \sum_{t} \text { utility } y_{r_{i}}(d, t)}$.

The variable utility $_{r_{i}}(d, t)$ is the reward agent $d$ has accumulated on route $r_{i}$ up to time $t$. Therefore an important factor is how often and in which intervals the heuristic is updated. This is especially relevant because the reward depends on the other agents. Using this simple model, we have performed experiments varying the frequency of heuristic adaptation. To prevent that all agents update their heuristics during the same round, each agent adapts with a given probability.

In order to model the imperfectness of information, we follow [1] who represent the traffic conditions as $L$ discrete states. We also consider $K$ information types. For each combination of state and information type, there is a cost function which also depend on the traffic volume. Thus, the dynamics on the routes are simulated according to these abstract cost functions which nonetheless can reproduce the macroscopic behavior of the system given that it includes a stochastic component regarding the traffic states.

The cost functions we use were adapted from [5], from which we also use much of the nomenclature, cost functions, and some scenarios, althought these were slightly modified to include the drivers's adaptation to route choice. We also assume $R$ alternative routes between two points in the network. Both have a traffic capacity of $M$ vehicles. Traffic can be in one of $L$ states (e.g. if $L=2$ we can have congested/non-congested states only). In the model, each state occurs with probability $q^{l}$. The control center predicts traffic based on imperfect information and provides information to the drivers which is also imperfect. There can be $K$ types of information. At a given time, one information $k$ is given with probability $p^{k}$. There can be any correspondence between $K$ and $L$ but mostly it is assumed that $L \geq K$, $K \geq 2$, and $L \geq 2$.

The probability of a state $l$ to occur after information $k$ is given by $\pi^{k, l}$. If $\pi^{k, l}=1 / L$, the information conveys basically no meaning i.e. it is tantamount to no information. When $L=K$ (i.e. for each $k$ there is an exclusive $l$ ) we can have perfect information provided one of the $\pi$ 's is one and the others are zero. For example, if $K=L=2$, when $\pi^{k, l_{1}}=1$ and $\pi^{k, l_{2}}=0\left(l_{1} \neq l_{2}\right)$, this is a situation of perfect information provision because it is known for sure which state is expected to occur after the information is provided.

\subsection{Parameters and Settings}

In the examples discussed here, $R=K=L=2$, and the travel cost functions used are linear of type $c_{i}^{l}=\zeta_{i}^{l}+v_{i}^{l} \times x_{i}^{k}$, where $x$ is the number of drivers. In particular we use the following functions [5]:

$\begin{array}{ll}c_{1}^{1}=1.0+0.6 \times 0.001 \times x_{1}^{k} & c_{1}^{2}=1.5+1.6 \times 0.001 \times x_{2}^{k} \\ c_{1}^{2}=1.0+0.4 \times 0.001 \times x_{1}^{k} & c_{2}^{2}=1.5+0.1 \times 0.001 \times x_{1}^{k}\end{array}$

The meaning of these functions is that the marginal costs $\left(v_{i}^{l}\right)$ of each route $r_{i}$ differ. Therefore we expect the number of drivers to be smaller in route 2 when $l=1$. In [5] the system equilibrium (without any toll etc.) is computed for $M=3000$ drivers: 2391.5 (route 1 ) and 608.5 (route 2) for $k=1 ; 1927.3$ (route 1) and 1072.7 (route 2) for $k=2$.

We show here that this equilibrium is never achieved when drivers use greedy strategies such as selection of route based 
on average of past travel time. However, when the selection is based on an utility function which includes the toll paid or the amount reimbursed, then traffic volumes are close to the system equilibrium.

\subsection{Scenarios}

In scenario I we are interested in reaching the equilibrium by only allowing users to apply their probabilities to select route $r_{i}$, given the information provision $k$. In our case this is done via adaptation of these probabilities (denotated as $\rho_{r, k}^{d}$ ) given the past utilities which are function of the costs. The basic mechanism for each driver $d$ is given by: $\rho_{r, k}^{d}=\frac{\overline{U_{r, k}^{d}}}{\sum_{i=1}^{R} \overline{U_{i, k}^{d}}}, 1 \leq i \leq R$, where $\overline{U_{r, k}^{d}}$ is the average of past utility for selecting route $r_{i}$ when the information provided was $k$.

In scenario II a toll is computed by the traffic control center and paid by the driver. This computation depends on the information $k$ so that drivers have to make a relation to their previous choice for the last situation where $k$ was provided. The toll value for each driver on route $r_{i}$ is calculated as $\tau_{r_{i}}^{k}=\frac{x_{r}^{k}-x_{r}^{k^{*}}}{x_{r}^{k}}$ where: $x_{r_{i}}^{k^{*}}$ is the number of drivers in the equilibrium situation for route $r_{i}$ given information $k$, and $x_{r_{i}}^{k}$ is the expected number of drivers, estimated by the last time $k$ was provided. Notice that this value can be positive (driver pays) or negative (driver gets a reimbursement).

The reasoning of the driver $d$ is as follows. If information $k$ is provided and the last time this has happened I was reimbursed because I have chosen route $r_{i}$, then I better select $r_{i}$ again with probability $\rho_{r_{i}, k}^{d}=1-$ rate $_{c u r}$. However, if information $k$ is provided and the last time this has happened I had to pay, then I select $r_{i}$ with probability $\rho_{r_{i}, k}^{d}=\tau_{r_{i}, k}^{d}$. Here rate $_{\text {cur }}$ is the rate of curiosity, i.e. a probability of $d$ experimenting another route (other than $r_{i}$ ) even though $r_{i}$ was good the last time; and $\tau_{r_{i}, k}^{d}$ is the toll paid by driver $d$ when selecting route $r_{i}$ under information $k$.

\subsection{Results}

We have used $M=3000$ agents in order to compare the results to those in [5]. If we let the drivers select routes only according to the utility they perceive (scenario I), then an user stable state is reached, but it is far from the system optimum. Number of drivers in both route (graphic not shown here) are not as mentioned before (Section 3.1). For $k=2$ the $M=3000$ drivers select each route with equal probability, so that nearly 1500 end up in each route, at any given time. This happens because the utility of drivers is nearly the same when we substitute $x_{i}^{k}=1500$ in the cost functions. For $k=1$, the expected system equilibrium is two-thirds in route 1 and one-third in route 2 . However, this equilibrium is nerver reached: users are stuck in a suboptimal stable state. Thus, a mechanism is needed which internalizes the externalities caused by the drivers selections.

Our scenario II simulates exactly what happens when drivers update their route choice probabilities according to a utility which is based on the past toll. Figure 1 depicts the number of drivers in each route, for each $k$, when a toll is charged and the curiosity rate is zero, meaning that drivers act only based on the the reward or punishment represented by the toll in the last time information $k$ was provided. As we see in that graph, the user equilibrium is now much closer to the system equilibrium. For $k=1$ the distribution of

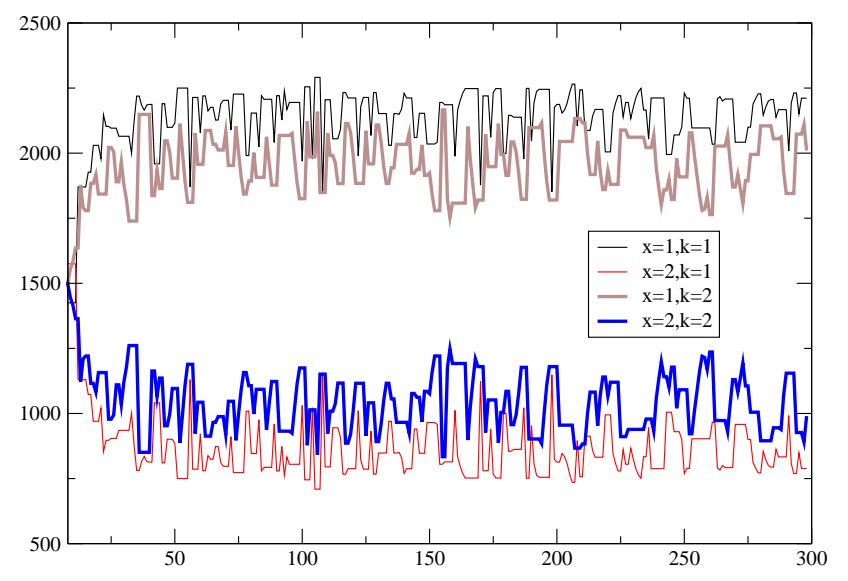

Figure 1: Number of drivers at each route, for each $k$; with toll and curiosity rate equal 0

drivers is around 2300 to 700 (routes 1 and 2 respectively), and for $k=2$ this distribution is around 2000 to 1000 .

Different rates of curiosity of course change these figures. The higher the rate, the more drivers deviate from the system equilibrium.

In summary, this paper shows that congestion tolls are useful to internalize the costs drivers impose to others when they act greedly. Tolls are the equivalent of the utility alignment proposed in the COIN framework, but departing from the assumption that agents are cooperative. For the context of multiagent systems, the present work can contribute to the development of a more general method of mechanism design, specially when agents are self-interested and the system optimum has to be reached without imposing explicit central coordination.

\section{REFERENCES}

[1] Arnott, R., de Palma, A., and Lindsey, R. Does providing information to drivers reduce traffic congestion? Transportation Research A 25 (1991), 309-318.

[2] Bazzan, A. L. C., And KlüGL, F. Case studies on the Braess paradox: simulating route recommendation and learning in abstract and microscopic models. Transportation Research C 13, 4 (August 2005), $299-319$.

[3] Klügl, F., And Bazzan, A. L. C. Route decision behaviour in a commuting scenario. Journal of Artificial Societies and Social Simulation 7, 1 (2004).

[4] Klügl, F., Bazzan, A. L. C., And Wahle, J. Selection of information types based on personal utility - a testbed for traffic information markets. In Proceedings of the Second International Joint Conference on Autonomous Agents and Multi-Agent Systems (AAMAS) (Melbourne, Australia, July 2003), ACM Press, pp. 377-384.

[5] Kobayashi, K., And Do, M. The informational impacts of congestion tolls upon route traffic demands. Transportation Research A 39, 7-9 (August-November 2005), 651-670.

[6] Tumer, K., And Wolpert, D. Collective intelligence and braess' paradox. In Proceedings of the AAAI (2000), AAAI Press, pp. 104-109. 